\title{
OpenFlow-Control of an OAM-Based Two-Layer Switch Supporting 100Gb/s Real Data-Traffic
}

 \\ M. Lavery ${ }^{(3)}$, F. Cugini ${ }^{(1)}$, M. Sorel ${ }^{(3)}$, A. Bogoni ${ }^{(2)}$ \\ (1) CNIT, Via Moruzzi 1, 56124 Pisa, Italy \\ (2) Scuola Superiore Sant'Anna, Via Moruzzi 1, 56124 Pisa, Italy \\ (3) University of Glasgow, Oakfield Avenue, Glasgow G12 8LT, UK, \\ mirco.scaffardi@cnit.it \\ Abstract: A two-layer orbital angular momentum and wavelength based switch is presented and \\ characterized up to $100 \mathrm{~Gb} / \mathrm{s}$ with coherent polarization-multiplexed real data traffic. The switch is \\ successfully reconfigured by applying an OpenFlow based SDN control plane. \\ OCIS codes: (060.4253) Networks, circuit-switched; (060.4259) Networks, packet-switched
}

\section{Introduction}

The orbital angular momentum (OAM) of the light can be exploited as switching domain together with wavelength to implement multi-layer switches, which promise a potential increase of the switch scalability [1].

Here we demonstrate for the first time the application of an automated SDN control system based on OpenFlow to a two-layer multi-port OAM-wavelength switch based on integrated concentric OAM modulators. A Matlabbased software is designed and interfaced with an OpenFlow agent, which drives the OAM switch configuration according to the OpenFlow commands. The switch is validated through $100 \mathrm{~Gb} / \mathrm{s}$ polarization-multiplexed (pol-mux) real data traffic. Dynamic reconfiguration with $10 \mu$ s switching time over 4 OAM modes is proved.

\section{Two-layer OAM-wavelength based switch architecture}

The OAM-wavelength based switch architecture is shown in Fig. 1 (a). The switch has a total number of $M \times N$ optical input and output ports ( $N$ subsets of $M$ ports). The ports of the same subset are addressed by the wavelength domain, while the different sets of ports are addressed by the OAM domain. The two mains elements of the switch are the OAM multiplexer (OAM mux), composed by integrated concentric OAM modulators [2,3], and the OAM demodulator/demultiplexer (OAM demod/demux), which can be implemented with two passive cascaded refractive elements [4]. Each port accepts a signal within the allowed set of $M$ wavelengths $\left(\lambda_{1}, \ldots \lambda_{\mathrm{M}}\right)$. For each input port, an OAM modulator converts the signal (phase front having a Gaussian spatial distribution) onto an OAM mode, i.e. with a phase front with an helical spatial distribution, of order $l$ among a set of $N$ OAM modes (OAM 1, ..,OAM N) depending on the targeted output ports subset. The order of the converted signal is set with a control signal (Ci) by thermal tuning [5]. The OAM demod/demux spatially separates OAM beams of different order $l$ and converts them to Gaussian. The different wavelengths are separated by means of an arrayed waveguide grating (AWG).

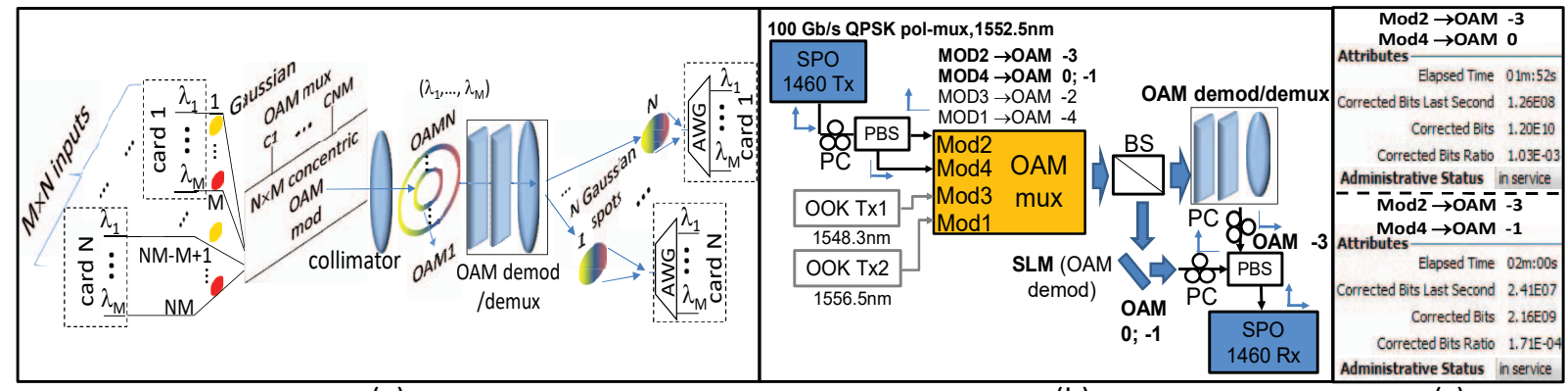

(a)

(b)

(c)

Fig. 1: (a) Two-layer (OAM and wavelength) switch architecture. (b) Setup for OAM switch characterization (100Gb/s QPSK real data traffic). PBS: polarization beam splitter; BS: beam splitter. (c) Screenshot of the card software interface in two different switch configurations.

\section{Real-data traffic characterization}

The proposed OAM-wavelength switch has been implemented and characterized exploiting $100 \mathrm{~Gb} / \mathrm{s}$ pol-mux real data traffic according with the setup shown in Fig. 1 (b). An OAM mux composed by four integrated concentric OAM emitters [3] has been exploited. The $100 \mathrm{~Gb} / \mathrm{s}$ coherent pol-mux traffic at $1552.52 \mathrm{~nm}$ is generated by a commercial network card (Ericsson SPO 1460) and split into orthogonal polarizations coupled to OAM Mod2 and OAM Mod4 respectively. After the OAM mux, the beams are split and sent to the OAM demux to demodulate the signal from OAM Mod2 and to a programmable spatial light modulator (SLM) to demodulate the signal from OAM Mod4. The signals are then coupled with orthogonal polarization and sent to the receiving card. OAM Mod 1 and 
OAM Mod 3 are fed with 30 Gbaud on-off keying (OOK) signals at 1556.5 and $1548.3 \mathrm{~nm}$ respectively. Fig. 1 (c) shows the screenshot of the card software interface in two different OAM switch configurations: OAM Mod4 set to OAM order $l=0$ and $l=-1$. In both cases the system recovers all the errors, demonstrating the operability with realtraffic equipment after OAM switching.

\section{OpenFlow-based OAM switch control demonstration}

The OpenFlow control of the switch architecture in Fig. 1 (a) has been implemented with the setup in Fig. 2 (a). A personal computer (PC) running the OpenFlow-driven control programs is connected to a tunable laser (TL) for setting the signal wavelength and a waveform generator for setting the proper voltage to the Mod4 emitter of the OAM mux. In order to demonstrate the OAM switch reconfiguration time, the OAM mux output signal is split and sent to the refractive elements based OAM demod/demux followed by an optical fibre positioned in correspondence of the demodulated OAM 0, and an SLM reconfigured to demodulate OAM -2 and -4. The OAM demod/demux and SLM outputs are coupled and sent to a real time oscilloscope. SLM and oscilloscope are controlled by the PC. A Matlab code has been implemented to send the proper commands from the PC when an OpenFlow request of switch reconfiguration occurs.

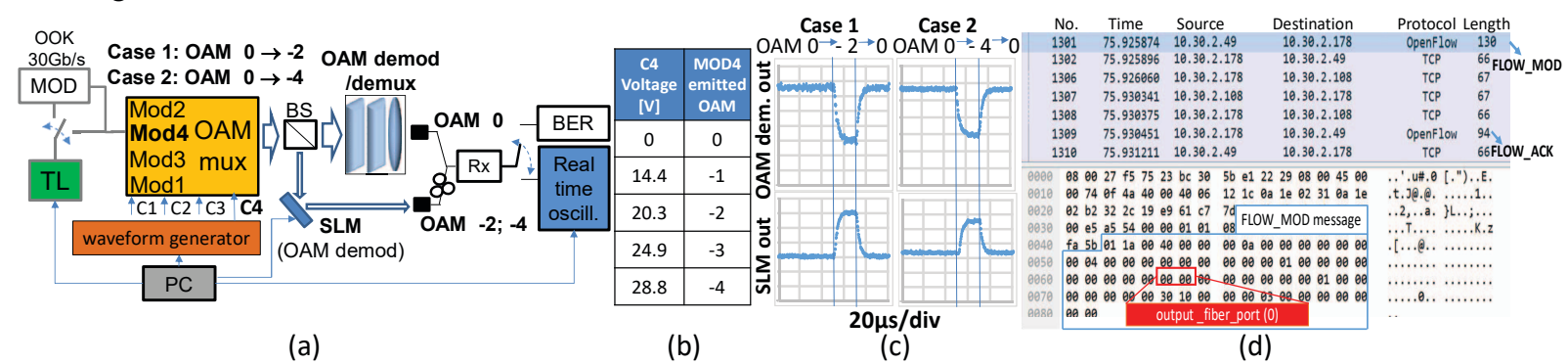

Fig. 2: (a) Setup for OAM-wavelength switch OpenFlow control. (b) Mapping of voltage applied on control input C4 and Mod4 emitted OAM order. MOD: modulation stage; BS: beam splitter; PC: personal computer; RX: receiver. (c) Intensity measured at the real time oscilloscope for the OAM demod output (top) and SLM output (bottom). Left: Mod4 switched from OAM 0 to -2 . Right: Mod4 switched from OAM 0 to -4. (d) OpenFlow capture at the OF Agent (IP 10.30.2.178), receiving FLOW MOD from the SDN Controller (IP 10.30.2.49) and triggering switching at PC (IP 10.30.2.108). FLOW_ACK is sent back after the switching is completed.

The sequence of operation is as follows: setting of the TL, setting of the SLM to demodulate the switched OAM mode (OAM -2 in case 1 and OAM -4 in case 2), setting of the waveform generator driving the OAM mux (Mod4). The table in Fig. 2(b) shows the mapping of the voltage applied to the control C4 and the OAM order emitted by Mod4. Fig. 2 (c) shows the oscilloscope traces during the switch reconfiguration in two cases, i.e. transition from OAM 0 to -2 (Fig. 2 (c)-left) and transition from OAM 0 to -4 (Fig. 2 (c)- right). The switching time is about $10 \mu \mathrm{s}$ in both cases. When data are transmitted through the switch $(30 \mathrm{~Gb} / \mathrm{s}$ OOK), error-free performance is measured at the switch output when the transition is ended. Fig. 2 (d) shows the collected OpenFlow message exchange with the SDN Controller (implemented by enhancing the proprietary Controller used in [6]). The overall switching time, including the SDN-based OAM switch controlling system, is successfully completed in less than $6 \mathrm{~ms}$.

\section{Conclusions}

We demonstrated a two-layer switch exploiting OAM and wavelength as switching domain, based on a single integrated active device for OAM modulation and multiplexing. The switch is tested with $100 \mathrm{~Gb} / \mathrm{s}$ coherent pol-mux real data traffic, showing proper operation in different switch configurations. The switch potential in a network scenario is demonstrated by applying a SDN control based on the OpenFlow protocol for reconfiguring the switch in two different cases. The dynamic measurements show $10 \mu$ s switching time and error-free operation. The proposed switch is suitable in data-centers where both electronic and optical circuit switching for long-live traffic coexist.

Acknowledgment. This work has been funded by the Project ROAM (contract number: 645361). The authors acknowledge support from the technical staff of the James Watt Nanofabrication Centre at Glasgow University. Thanks to Francesco Fresi and Bilal Hussain for support.

6. References

[1] M. Scaffardi et al., “A Silicon Microring Optical 2x2 Switch Exploiting Orbital Angular Momentum for Interconnection Networks up to 20Gbaud”, IEEE J. Light. Tech. 35, 3142-3148 (2017).

[2] X. Cai, et al.,"Integrated compact optical vortex beam emitters", Science 338, 362-366 (2012).

[3] M. Scaffardi et al., "Tunable Orbital Angular Momentum (OAM) Conversion on 100Gb/s Real Data Traffic by Exploiting Concentric Waveguide Emitters”, in Proc. ECOC'17, P2.SC5.4 (2017).

[4] M.P.J. Lavery et al., "Refractive elements for the measurement of the orbital angular momentum of a single photon" Opt. Exp. 20, $2110-2115$ (2012).

[5] M.J. Strain, et al., "Fast electrical switching of orbital angular momentum modes using ultra-compact integrated vortex emitters," Nature Comm. 5, $4856(2014)$.

[6] F. Paolucci, et al., "Multipath restoration and bitrate squeezing in SDN-based elastic optical networks", Journal of Photonic Network Communications. 28, 45-57 (2014). 